\title{
Light absorption by d-dimensional organic semiconductors under exciton transitions between broad bands
}

\author{
N. I. Grigorchuk \\ Institute of Semiconductor Physics, National Academy of Sciences of Ukraine, Kyiv, 252028, Ukraine
}

\begin{abstract}
Within the framework of the dipole approximation the line-shape of light absorption for exciton transitions between broad bands in one-, two- and three-dimensional organic semiconductor structures are calculated. Exciton damping due to lattice imperfections is accounted for as a frequency independent parameter. The obtained analytical expressions allow analyses of the lineshape for different space dimensions of structure depending on bandwidth difference, damping parameter and temperature.
\end{abstract}

Keywords: low-dimensional organic crystals, light absorption, exciton transitions.

Paper received 01.03.99; revised manuscript received 02.04.99; accepted for publication 19.04.99.

\section{Introduction}

Various organic semiconductors (OS) have been a subject of intensive studies for many years. Due to the number of physical effects and their potential applications in electronic and optical devices in newly available materials [1]-[3] there has been increasing interest in the investigation of organic structures of low dimensionality [4][6].

It is well known [7] - [8] that in contrast to the atomic spectra (or spectra of simple molecules) the spectra of orqanic compounds contain the discrete lines along with the broad bands $\left(\sim 10^{2}-10^{3} \mathrm{~cm}^{-1}\right)$. The nature of these bands attracts the attention of researchers during four last decades.

Using a method of selective laser excitation, Personov has shown that at rather low temperatures the broad bands are caused by a non-uniform broadening and in them the huge number of narrow no-phonon lines is hidden. At the same time it was observed [7] that some spectra of the organic compounds embedded in frozen matrices of $n$-paraffins, remain a broadband even at sufficiently low temperatures and are not split on numbers of fine lines as one might expect from results of [9].

As a rule, the band broadening in the absence of an external action or dephasing processes is connected with the scattering of excitons on phonons, impurities, dislocations and other lattice imperfections reducing the lifetime of exciton. For the perfect OS one of basic mechanisms of the exciton energy losses usually comes from their interaction with phonons. Especially, it seems, the multi-phonon processes may play considerable role in the formation of broad bands. However, phonons not always can give the appreciable contribution to the formation of the broad bands in organic structures [10]. At low temperatures the broad band could be expected also due to inhomogeneous broadening of close located no-phonon lines, but for absorption the contribution of such mechanism can practically be neglected [11].

There arise a problem of interpretation of broad bands in OS, which are not split on separate lines at low temperatures and not broaden with temperature rising.

An attempt to examine this problem for $3 D$-crystals as well as for the case of narrow exciton bands was undertaken by us earlier in [12-13]. We treated the simple twoband model, in which the distribution of excitons over sublevels of the lowest band was considered as established, and their photo-excitation to a higher band was studied. This looks like a two-photon problem [14-15], but differs only by the fact, we consider the 1st exciton band as a band of thermalized exciton states. This work represents an extension of techniques previously [12] applied to the bulk materials.

In this paper, we apply the above stated approach to calculate the absorption in OS of low spatial dimensional- 


\section{N. I. Grigorchuk: Light absorption by d-dimensional organic ...}

ity for another marginal case of broad exciton bands. Using the model mentioned above we have calculated here the contour shape of light absorption for one-, two-, and threedimensional $(1 D, 2 D$ and $3 D)$ periodic OS. We also have lead comparison the line shapes of light absorption in OS of different dimensionality.

The rest of the paper is arranged as follows. In Sec. 2 we shortly describe our working model and the tasks considered. In Sec. 3 we present the basic formulae for calculation. Section 4 is devoted to the discussion of results and Sec. 5 to the conclusions.

\section{Model and main assumptions}

We study a periodic OS with simple nondegenerate bands whose extrema are located at the center of the Brillouin zone. Every elementary cell contains only one molecule. We assume that the exciton bands are sufficiently separated and that the configuration mixing of exciton states in each band is absent. The excitons are supposed to be capable to perform a Bloch-wave-like coherent motion. In the media with the large dielectric constant exciton motion can be described by means of the effective mass approximation [16]. According to this approach, the energy of an exciton in the lowest band may be written down as follows

$$
\varepsilon_{i}\left(k_{i}\right)=-\sum_{j=1}^{D}\left(B_{i j}-\frac{\hbar^{2} k_{i j}^{2}}{2 m_{i j}}\right), \quad i=1,2,
$$

where $\left\{k_{i 1}, k_{i 2}, \ldots, k_{i D}\right\}$ are the components of the exciton quasi-momentum vector $\mathbf{k}_{i}$ in the $D$-dimensional space, $B$ is the bandwidth of the $i$-th band in the $j$-th direction, and $m_{i j}$ is the exciton mass. $m_{i j}$ is connected with the band parameter $B_{i j}$ by means of the relation: $B_{i j}=\hbar^{2} / m_{i j} a_{j}^{2}$, where $a_{j}$ is the lattice constant. Using Eq. (1) for $\varepsilon_{i}\left(k_{j}\right)$, the distribution function of excitons over sublevels of the first exciton band one may to express in the form

$$
W\left(k_{1}\right)=\left(\frac{2 \pi \hbar^{2}}{k_{B} T}\right)^{D / 2} \prod_{j=1}^{D} \frac{1}{n_{j} a_{j} \sqrt{m_{i j}}} e^{-\frac{\hbar^{2}}{2 k_{B} T}\left(\frac{k_{1 j}^{2}}{m_{1 j}}\right)},
$$

where $n_{j}$ is the number of knots in $j$-th direction and $T$ is the temperature. Hence, in case of broad bands for any dimensionality of OS, the excitons in the 1 st band can be considered as ordinary gas obeying usual Boltzmann statistics.

The transition matrix element from the initial $\left|f_{1} \mathbf{k}_{1}\right\rangle$ to the more higher quantum state $\left|f_{2} \mathbf{k}_{2}\right\rangle\left(f_{i}\right.$ describes some other quantum numbers), in the dipole approximation look similarly to that one derived in [12]. The dipole approximation is valid provided the exciton coherence length is much less than the wavelength in organic structure of the absorbed photon. We will neglect the photon momentum as an infinitesimal value comparing with any other pulses in the system. Then for transition frequency we get $\omega_{f_{2} f_{1}} \cong \Omega-\sum_{j=1}^{D} \delta B_{j}\left[1-k_{1 j}^{2} a_{j}^{2} / 2\right]$,

where $\Omega$ is the energy distance between the bottoms of the first and second exciton bands.

Averaging the matrix element over an initial states, which are populated with the statistical weight given by Eq. (2), and summing over all final ones, one finds the probability of transition between examined states. This probability is directly proportional to the dimensionless frequency function [12]

$$
\begin{aligned}
& F(\omega)=\Omega_{D}\left(\frac{\hbar^{2}}{2 \pi k_{B} T}\right)^{D / 2} \times \\
& \times \Re \int_{0}^{\infty} d t e^{i t \varepsilon_{D}} \prod_{j=1}^{D} \frac{1}{m_{j} a_{j}} \int_{-\pi}^{\pi} d x e^{-x^{2}\left(A_{j}+i t \delta B_{j} / 2\right)}
\end{aligned}
$$

which in general form defines the line-shape of light absorption by excitons for such transitions. Here

$\Omega_{D}=\Omega-\sum_{j=1}^{D} \delta B_{j}, \quad \varepsilon_{D}=\omega-\Omega_{D}+i \Gamma / 2$,

$A_{j}=\hbar^{2} /\left(2 m_{1 j} a_{j}^{2} k_{B} T\right)$

The boundaries of the 1st exciton band are accounted by second integral of Eq.(4). The symbol $\mathfrak{R}$ means that the real part of the expression should be taken. Beyond the exponent of Eq. (4) we have omitted the terms of the order of magnitude of equal to, or even higher than the $\delta B / \Omega_{D}$. This corresponds to the assumption that the energy distance between the first and second exciton bands should much more exceed the difference of their width:

$\Omega \gg 2 D \delta B$.

The damping of the excitons due to phonons or lattice imperfections is accounted for by a frequency-independent parameter $\Gamma$. We also have assumed that

$\delta B / \Gamma \gg>1$,

i.e., we considered that the difference of two band width is much more than any possible damping in OS. It is necessary to keep in mind, that variable $t$ is dimensional variable (in contrast to another one, $x$ ) and is measured in seconds.

\section{Basic formulae}

Let us consider the temperatures for which $A_{j} \gg 1$. Then the integration limits in second integral of the Eq. (4) may be extended to infinity, and using the Poisson integral $\int_{-\infty}^{\infty} e^{-\alpha x^{2}} d x=\sqrt{\pi / \alpha}$, equation (4) can be presented as 


\section{N. I. Grigorchuk: Light absorption by d-dimensional organic ...}

$$
\begin{aligned}
& F(\omega)=\Omega_{D}\left(\frac{\hbar^{2}}{k_{B} T}\right)^{D / 2} \times \\
& \times \prod_{j=1}^{D} \Re \int_{0}^{\infty} d t e^{i t \varepsilon_{D}} \frac{1}{a_{j} \sqrt{m_{j} \delta B_{j}}}\left(i t+\frac{2 A_{j}}{\delta B_{j}}\right)^{-1 / 2}
\end{aligned}
$$

It is easy to see, that the integral of complex conjugate integrand is reduced to integral of Eq. (8) by changing $t \Rightarrow-t$. Formally, it allows the integral of Eq. (8) to be represented as a half of the integral in the limits from $-\infty$ up to $\infty$. Then the expression (8) may be considered as exponential Fourier transform. Determining the Fourier-image for the cases $D=1,2,3$, we receive then:

$$
\begin{aligned}
& \left.F(\omega)\right|_{D=1}=\Omega_{x} \sqrt{\pi \alpha_{x}} \Re \frac{e^{-\left|\alpha_{x} \varepsilon_{x}\right|}}{\sqrt{\varepsilon_{x}}}, \\
& \left.F(\omega)\right|_{D=2}=\pi \Omega_{x y} \sqrt{\alpha_{x} \alpha_{y}} \times \\
& \times \Re e^{-\frac{1}{2}\left|\left(\alpha_{x}+\alpha_{y}\right) \varepsilon_{x y}\right|} I_{0}\left[\varepsilon_{x y}\left(\alpha_{x}-\alpha_{y}\right)\right] \\
& \left.F(\omega)\right|_{D=3}=2 \Omega_{\| \perp} \sqrt{\pi} \alpha_{\perp} \sqrt{\alpha_{\|}} \times \\
& \times \Re \sqrt{\varepsilon_{\| \perp}} e^{-\left|\alpha_{\perp} \varepsilon_{\| \perp}\right|}{ }_{1} F_{1}\left[\frac{1}{2} ; \frac{3}{2} ;-\left(\alpha_{\|}-\alpha_{\perp}\right) \varepsilon_{\| \perp}\right],
\end{aligned}
$$

where

$$
\begin{aligned}
& \Omega_{k}=\Omega-\delta B_{k}, \Omega_{k l}=\Omega-\delta B_{k}-\delta B_{l}, \alpha_{k}=\frac{2 A_{k}}{\delta B_{k}}, \\
& \varepsilon_{k l}=\omega-\Omega+\delta B_{k}+\delta B_{l}+i \Gamma / 2, k, l=x, y, z ; \|, \perp,
\end{aligned}
$$

$I_{0}(x)$ is the modified Bessel function of zeroth-order with complex argument, ${ }_{1} F_{1}(a, b, z)$ is confluent gypergeometric function, which, in particular, at $a=1 / 2, b=3 / 2$ takes the form [17]:

$$
{ }_{1} F_{1}\left(\frac{1}{2}, \frac{3}{2},-x\right)=\frac{1}{2} \sqrt{\frac{\pi}{x}} \operatorname{erf} \sqrt{x},
$$

where erf $x$ is the probability integral. The formulae (9)(11) describe the shape of absorption bands for a general case of an anisotropic lattice: rectangular, as in the case of $2 D$-semiconductors, or with the allocated symmetry axis, as for $3 D$-case, for which the cylindrical symmetry was chosen for instance.

In the case, when we deal with the isotropic organic structure (the lattice has the square or cubic form) then in the previous formulae it is necessary to put

$\delta B_{k}=\delta B_{l} \equiv \delta B, \Omega_{D} \equiv \Omega-D \delta B, \quad \alpha_{j} \equiv \alpha$,

where $D$ describes, as well as above, the dimensionality of the OS. Next, taking in the Eq. (1) into consideration that

$\lim _{x \rightarrow 0} \frac{\operatorname{erf} x}{x}=\frac{2}{\sqrt{\pi}}$

we obtain

$\left.F(\omega)\right|_{D=1}=\Omega_{1} \sqrt{\pi \alpha} \Re \frac{e^{-\left|\alpha\left(\omega-\Omega_{1}+i \Gamma / 2\right)\right|}}{\sqrt{\omega-\Omega_{1}+i \Gamma / 2}}$,

$\left.F(\omega)\right|_{D=2}=\Omega_{2} \pi \alpha \Re e^{-\left|\alpha\left(\omega-\Omega_{2}+i \Gamma / 2\right)\right|}$,

$\left.F(\omega)\right|_{D=3}=2 \Omega_{3} \sqrt{\pi} \alpha^{3 / 2} \times$

$\times \Re \sqrt{\omega-\Omega_{3}+i \Gamma / 2} e^{-\left|\alpha\left(\omega-\Omega_{3}+i \Gamma / 2\right)\right|}$.

Thus, the formulae (16)-(18) with accounting of damping describe the band shape, which arise by light absorption in $1 D$-, $2 D$ - and in $3 D$ isotropic OS. This our result can be reached by another way, when, for example, at evaluating of the second integral in Eq. (4) the saddle-point integration is used. The most simple expressions for the band shape can be obtained in the absence of the damping $(\Gamma \rightarrow 0)$. They are easily appeared from Eqs. (16)-(18). In the other limit case, when the width of both bands coincides, i.e. $\delta B=0$, the absorption curve takes the form of symmetric Lorentz with the half-width of $\Gamma / 2$.

At last, extracting the real part from Eqs. (16)-(18) we obtain the expressions enabling to calculate immediately the line shape of absorption for various dimensionalities of OS:

$$
\begin{aligned}
& \left.F\left(x_{1}\right)\right|_{D=1}=\frac{\Omega_{1}}{\Gamma} \sqrt{2 \pi \gamma} \cos \gamma \frac{e^{-\left|\gamma x_{1}\right|}}{\left(x_{1}^{2}+1\right)^{1 / 2}} \times \\
& \times\left[\sqrt{\sqrt{x_{1}^{2}+1}+x_{1}}-\sqrt{\sqrt{x_{1}^{2}+1}-x_{1}} \tan \gamma\right],
\end{aligned}
$$

$\left.F\left(x_{2}\right)\right|_{D=2}=\frac{\Omega_{2}}{\Gamma} 2 \pi \gamma \cos \gamma e^{-\left|\gamma x_{2}\right|}$,

$\left.F\left(x_{3}\right)\right|_{D=3}=2 \frac{\Omega_{3}}{\Gamma} \sqrt{2 \pi \gamma} \gamma \cos \gamma e^{-\left|\gamma x_{3}\right|} \times$

$\times\left[\sqrt{\sqrt{x_{3}^{2}+1}+x_{3}}+\sqrt{\sqrt{x_{3}^{2}+1}-x_{3}} \tan \gamma\right]$. 


\section{N. I. Grigorchuk: Light absorption by d-dimensional organic ...}

In these expressions

$\gamma=\alpha \Gamma / 2$

$x_{1}=\frac{\omega-\Omega_{1}}{\Gamma / 2}, \quad x_{2}=x_{1}+2 \delta B / \Gamma, x_{3}=x_{1}+4 \delta B / \Gamma$.

The region of variable change for $x_{1}$ has restriction, which follows from the condition

$$
\tan ^{2} \gamma \leq \sqrt{\sqrt{x_{1}^{2}+1}+x_{1}} / \sqrt{\sqrt{x_{1}^{2}+1}-x_{1}} .
$$

For $x_{1}$ which do not obey the condition (24), the function $F\left(x_{1}\right)=0$. Analyzing expressions (19)-(21), it is possible to make a conclusion that with increase of the dimensionality $D$ of the OS the absorption band, as a whole, is shifted to the short-wave range of a spectrum proportionally to the value of $2 D \delta \mathrm{B} / \Gamma$.

In order to compare the shape of the absorption band for OS of different spatial dimensionalities, we bring in coincidence the origin of the different frequency scales, by setting

$x_{1}=x_{2}=x_{3} \equiv x$,

It means that we have chosen

$\Omega_{1} \approx \Omega_{2} \approx \Omega_{3} \equiv \Omega$.

Such approximation unsignificantly will affect relative intensity of lines, because in accordance with our previous assumption (6), the energy distances between the first and following exciton band much more exceeds width of the 1 st band in any of the $\mathbf{k}$-directions.

\section{Discussion of results}

Using the Eqs. (19)-(21), in Fig.1 (A, B) the curves of light absorption for different dimensionality of OS are plotted. All curves correspond to the same certain temperature which enters into parameter $\gamma$. The upper panel (A) of the Fig. 1 is consistent with $\delta B / \Gamma=8$, whereas its lower panel (B) corresponds to the value $\delta B / \Gamma=16$. Both parts of the figure clearly indicate that under band-to-band exciton transitions with increase of the dimensionality of OS, first of all, the long-wave wing of the absorption band is appreciably changed: the intensity of light absorption is increased and becomes the highest one (for any frequency of this wing) for $2 D$-semiconductors. For them, the maximum of intensity corresponds to the transition frequency $\omega=\Omega-4 \delta B$.

For both the $1 D$ and $3 D$-OS there is an additional shift of the maximum (besides that caused by the difference of the width of two bands) in the direction of the violet spectra range. For $3 D$-structure this shift somewhat exceeds one obtained in $1 D$-structure. At given $\delta B / \Gamma=8$, the relative intensities of lines are the highest ones for $2 D$-structure and become minimal for $3 D$-structure. If one chooses the value $\delta B / \Gamma$ twice large, then intensity of absorption gets highest

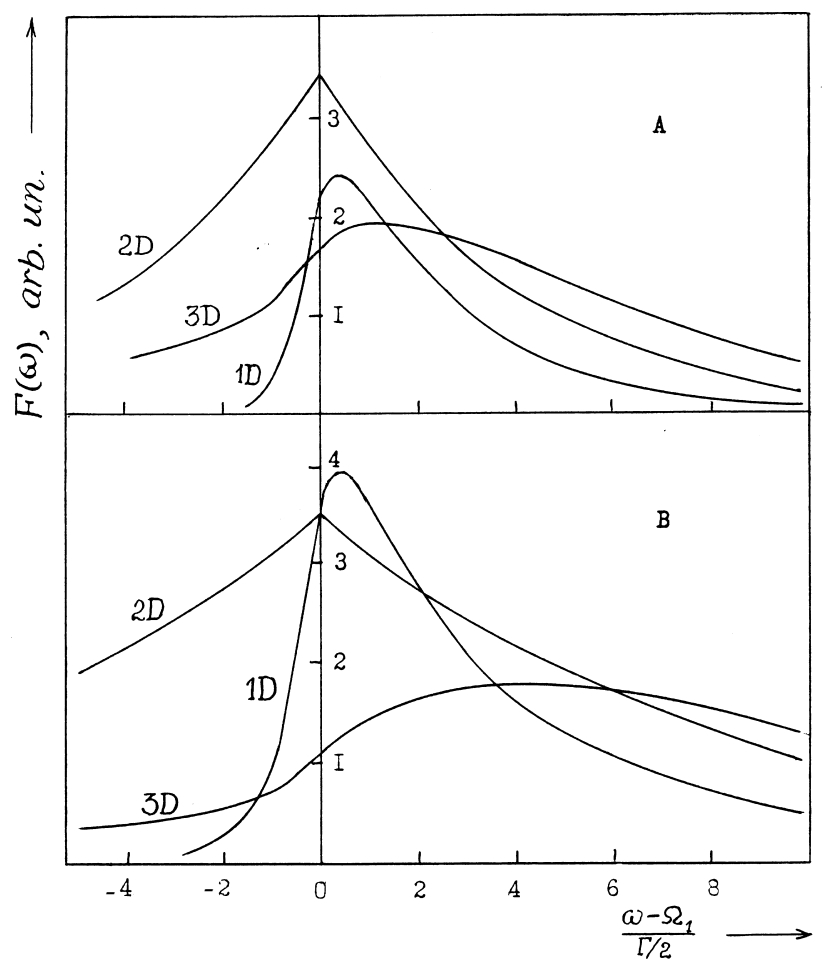

Fig. 1. The contour shape of light absorption under exciton transitions between broad bands for $1 D$-, $2 D$-, and $3 D$-dimensional organic semiconductors, $A=4: \delta B=8 \Gamma$ (panel $A) ; \delta B=$ $=16 \Gamma$ (panel $\mathrm{B})$.

for one-dimensional structure, and for three-dimensional one becomes even less (see lower panel of Fig. 1). Most simply the relative intensity of lines could be estimated for one of displayed frequencies. So, for example, at $x=0$, with increase of dimensionality of OS, it will be described by ratio of:

$(1-\tan \gamma): \sqrt{2 \pi \gamma}: 2 \gamma(1+\tan \gamma)$,

which are applicable both for arbitrary temperatures and for any ratio $\delta B / \Gamma$ satisfying the condition of Eq. (24). An enhancement of the ratio $\delta B / \Gamma$ at fixed temperature results, as it is easy to see from Eqs. (19)-(21), to reduction of $\gamma$; consequently, the line intensity is increased in one-dimensional case, whereas in two- and three-dimensional cases it decreases only. Moreover, in three-dimensional case this reduction is the most essential. Such tendency maintains also for frequencies close to the frequency of absorption peak and varies only on wings of absorption (see Fig.1). Comparing the absorption curves for the OS of different dimensions (for the same values of the assumed parameters), it should be noted that the line appreciably broadens for transitions from $1 D$ - to $2 D$-OS. The opposite situation, i.e., a narrowing the line occurs for the transition from the $2 D$ to the $3 D$ case. However, the half-width of the absorption curve 


\section{N. I. Grigorchuk: Light absorption by d-dimensional organic ...}

in the $3 D$ case remains somewhat larger than one in the $1 D$ case (see Fig. 1, A-, B-panels). The line broadening in the $2 D$ case may be explained by the fact that for normal light incidence the probability of the transfer of excitation between molecules in a plane increases compared to that in a linear chain proportionally to both the number of neighboring molecules and to the wave function overlap. However, in the bulk, this probability is decreased, and the absorption curve is narrowed almost to the width of the $1 D$ structure. This effect is caused by the decrease of the effective bandwidth difference, which may be due to the increase of the effect of exciton dynamics. Influence of the exciton motion on the line-shape of optical absorption was studied in more detail, for example, in [18] and results as a rule in line narrowing.

Generally, the line-shape is the product of two factors, the probability for optical transitions between the first and second bands and the Boltzmann distribution of the exciton kinetic energies. From Eqs. (19)-(21) it follows that the line-shape of absorption under exciton transitions between broad bands depends in the same measure both on the temperature and the width difference of two bands. Moreover, both factors act in the same direction. For instance, the decrease of the difference of $\delta B$ gives the same result as temperature lowering: the intensity of absorption falls. From the physical point of view, this is due to an equal probability for an exciton with the defined value of $\mathbf{k}_{1}$ to scatter on a phonon or an another exciton. The numerical evaluation for $3 D$-OS exhibits that twofold enhancement of the $\delta B$ or temperature, results in the decreasing in the intensity of the absorption as much.

This our result essentially differs from one obtained earlier [13] for the case of narrow exciton bands.

The well known mechanism of inhomogeneous broadening due to static disorder in the quasiperfect crystal, seems to play more important role for solutions [7]. An estimation may be done by comparing the values of the energy dispersion of one of the exciton levels with the band-width difference $\delta B$. The first one is found to be much less for the considered system.

The Eqs. (19)-(21) describe the line-shape in the case when the effective masses of an exciton $m_{1}$ and $m_{2}$ in the first and second bands have positive sings. When effective masses of an exciton are negative then the transition frequency, at $\mathbf{k}_{2}=\mathbf{k}_{1}$ becomes

$\omega_{f_{2} f_{1}} \cong \Omega+\sum_{j=1}^{D} \delta B_{j}\left[1-k_{1}^{2} a_{j}^{2} / 2\right]$,

hence, replacing in the Eq. (4) the integral over $d x$ on the integral

$$
i_{1}=\int_{-\pi}^{\pi} d y \exp \left\{+\left(A_{j}+i t \delta B / 2\right) y^{2}\right\}
$$

one gets the general expression for the line-shape of absorption with negative exciton masses. Further, it is easy to see that at replacement of variable $y \rightarrow \pi-x$, the integral $i_{1}$ of Eq. (29) passes in to the last integral of Eq. (4). If one takes into account also that

$\operatorname{Tr}\left\{e^{-\varepsilon_{1}\left(\mathbf{k}_{1}\right) / k_{B} T}\right\}=\frac{1}{Z} e^{3 B_{1} / k_{B} T}$,

(where $\mathbf{T r}$ is the trace, and $Z$ is the statistical sum) then it becomes clear that the expression for the function $F(\omega)$ keeps the former kind as in Eq. (4). This means that for negative exciton masses the shape of absorption curves will be defined by the same formulae (9)-(11). Such conclusion is clear from physical reasons as well: under simultaneous changes of the signs of the exciton effective masses in the first and in the second bands, in the case of direct transitions, the energy distances between bands is kept for each of wave vectors of Brillouin zone.

For many organic compounds which in experiment display preferentially the properties of individual molecules, our model is invalid. It seems it may hold for those OS which exhibit generally the crystalline properties that are associated with the coherent motion and the band structure [19]. To the last category one may attribute, for example, the low dimensional crystal structure of 1,2, 4, 5-thetrachlorbenzene, 1,4-dibromnaphthalene, etc. For such OS there are both numerous experiments (e.g., [20]) and numerical calculations (e.g., [21]) studying the light absorption due to the exciton transitions from the ground state to the singlet or triplet exciton bands. But we are not aware of any data on the exciton absorption caused by transitions between exciton bands in these OS. Moreover, we are not aware of any experiments on transformation of the absorption line-shape with changing dimensionality of the same material.

\section{Conclusions}

Surmising, we can conclude that within the framework of the two-band model the additional nonthermal broadening mechanism arises owing to the difference between the density of states of the bands under consideration.

In the case of broad exciton bands, the difference of their widths and temperature of MC are two main comparable factors responsible both for the contour shape and for the intensity of absorption. The increase of band difference gives the same result as an enhancement of the temperature of OS: the intensity of light absorption decreases, and the absorption maximum shifts to the short-wave spectrum range.

The absorption contour essentially broadens at transitions from $1 D$ - to $2 D$-OS and is narrowed appreciably at transition from 2 to $3 D$-dimensionality. Thus, there is an additional shift to the violet spectra range due to the difference of widths of two bands. This shift is proportional to the dimensionality of the structure.

Simultaneous changing of the signs of the effective masses does not change the form of an absorption contour.

If the state of the lowest band are evenly filled by excitons, one can study the states density of higher-lying bands.

Benchmark applications to nonlinear exciton-tobiexciton absorption are anticipated. 


\section{N. I. Grigorchuk: Light absorption by d-dimensional organic ...}

\section{References}

1. T. Tokihiro, Y.Manabe, and E. Hanamura, Superradiance of Frenkel Excitons in Linear Systems // Phys. Rev. B, 47(4), pp.20192030 (1993).

2. Y. Shimoi and S. Abe, Theory of Triplet Exciton Polaron and Photoinduced Absorption in Conjugated Polymers // Phys. Rev. $B, 49$ (20), pp. 14113-14121 (1994).

3. L.Rossi, G. Lanzani, and F. Garnier, Charged Photoexcitations in Thiophene-based Molecular Semiconductors // Phys. Rev. B, 58(11), pp. 6684-6687 (1998).

4. A. Tomioka, and K. Miyano, Numerical Study of Excitons in a Two-Dimensional Organic Dye Aggregate // Phys. Rev. B, 54(5), pp. 2963-2967 (1996).

5. N.T. Harrison, G.R. Hayes, R.T. Philips, and R.H. Friend, Singlet Interaction Exciton Generation and Decay in Poly (p-Phenylenevinylene) // Phys. Rev. Lett., 77(9), pp. 1881-1884 (1996).

6. S. Abe, M. Schreiber, W.P. Su, and Y. Yu, Excitons and Nonlinear Optical Spectra in Conjugated Polymers // Phys. Rev. B, 45(16) pp. 9432-9435 (1992).

7. Spectroscopy and Excitation Dynamics of Condensed Molecular Systems, ed. by V.M. Agranovich and Hochstrasser, NorthHolland, Amsterdam (1983).

8. V.L. Broude, E.I. Rashba, and E.F. Sheka, Spectroscopy of molecular excitons, Energoizdat, Moskow (1981).

9. R.I.Personov, Selective spectroscopy of a complex molecules in solutions and its application, Preprint of Institute of Spectroscopy (USSR), No.14, Troitsk (1981).

10. M. Higuchi, T. Nakayama, and N. Iton, Studies of Higher Triplet Exciton Bands through T-T Absorption in Naphthalene Single Crystal // J. Phys. Soc. Jpn. 40(6), pp. 250-257 (1976).

11. O.N. Karataev, T.M. Naumova, V.P. Karpov and M. Ju. Kalitievskii, Bandwidth and Shape of Bands under T-T Absorption, in «Modern Problems of Spectroscopy of crystals», Naukova
Dumka, Kyiv (1976), pp. 188-195.

12. N. I. Grigorchuk, L. G. Grechko, Lineshape of Light Absorption by Thermalized Frenkel Excitons under Band-to-Band Transitions // Phys.Stat.Sol.(b), 153(2), pp. 633-640 (1989).

13. N. Grigorchuk, Line-Shape of Light Absorption by Excitons in One-, Two- and Three-Dimensional Molecular Structures // Solid State Commun.,100(12), pp. 861-864(1996).

14. F. X. Bronold and A. R. Bishop, Nonlinear Optics of Conjugated Polymers: A coupled Exciton-Phonon Gas Approach // Phys. Rev. B, 53(20), pp. 13456-13476(1996).

15. A. Shimizu, T. Ogawa, and H. Sakahi, Two-Photon Absorption Spectra of Quasi-Low-Dimensional Exciton Systems // Phys. Rev. $B, 45(19)$, pp. 11338-11341 (1992).

16. D. P. Craig, and S. H. Walmsley, Excitons in Molecular Crystals. Theory and Applications. Benjamin, New York, Amsterdam, (1968).

17. M. Abramovitz and I. Stegun, Handbook of Mathematical Functions, Dover, New York (1972).

18. B. N. J. Person, F. M. Hoffman, and R. Ryberg, Influence of Exciton Motion on the Shape of Optical Absorption Lines: Applications to Vibrations at Surfaces // Phys. Rev. B, 34(4), pp.2266-2283(1986).

19. H.Haken, and P. Reineker, The Coupled Coherent and Incoherent Motion of Excitons and its Influence on the Line Shape of Optical Absorption // Zeitschrift Phys. 249(1), pp. 253-268(1972).

20. D.M. Burland, U. Kunzelmann, and R.M. MacFarlane, Exciton Scattering Processes in $(1,4)$-Dibromnaphthalene // J. Chem. Phys. 67(5), pp. 1926-1934 (1977).

21. M.Schreiber, and Y. Toyazawa, Numerical Experiments on the Absorption Lineshape of the Exciton under Lattice Vibrations. I. The Overall Lineshape // J. Phys. Soc. Jpn. 51(5), pp. 1528-1536 (1982). 